\title{
The effects of changes in estradiol and progesterone concentrations during the menstrual cycle on resting metabolic rate
}

\author{
S. E. Hillier ${ }^{1}$, M. C. Venables ${ }^{1,2}$ and R. P. Craven ${ }^{1}$ \\ ${ }^{1}$ Functional Food Centre, Oxford Brookes University, OX3 OBP, UK and ${ }^{2}$ Human Health and Performance Research \\ Group, Oxford Brookes University, OX3 OBP, UK
}

There is some debate as to whether resting metabolic rate (RMR) fluctuates during different phases of the menstrual cycle as a result of changes in estradiol and progesterone concentrations ${ }^{(1,2)}$. One reason for this could be the failure to establish accurate menstrual phase definition through biochemical analysis of hormone concentrations. The aim of this study was to investigate RMR at three set time points during the menstrual cycle.

Venous blood samples for baseline measurements of estradiol and progesterone were collected every other week day from eight naturally cycling women for one complete menstrual cycle; age 28 (SD 3) years, weight 62.04 (SD 9.75) kg and height 1.69 (SD 0.09 ) m. Indirect calorimetry using a ventilated hood system (Deltatrac, Datex Instrumentarium Corp., Helsinki, Finland) to calculate RMR was performed on three test sessions during one complete menstrual cycle. In the $24 \mathrm{~h}$ period prior to each RMR measurement participants replicated their diet and avoided exercise, caffeine and alcohol.

Venous blood samples were taken before each test session to confirm estradiol and progesterone levels. Participants were supine for 30 min to allow initial stabilisation and acclimatisation to the canopy and instrument noise. Respiratory gas exchange data were then collected for a further $30 \mathrm{~min}$ and an average RMR was calculated. Data are presented as means (SD). Variables were analysed using oneway repeated measures ANOVA. Significance was accepted at the $P<0.05$ level.

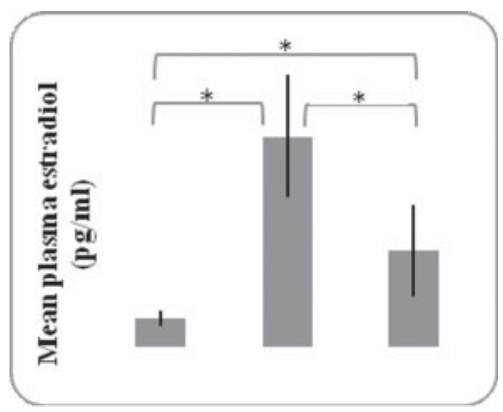

Fig. 1. Plasma estradiol concentration.

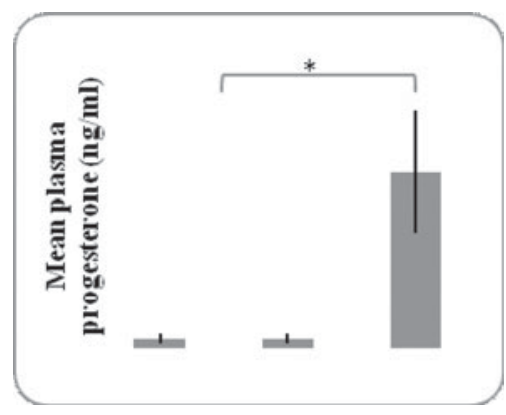

Fig. 2. Plasma progesterone concentration.

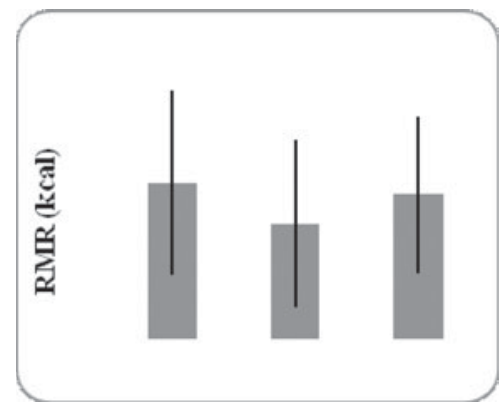

Fig. 3. RMR

*Significant difference between test sessions. $M$, menstruation; $F$, follicular; $L$, luteal.

Hormone concentrations differed significantly during one complete menstrual cycle (estradiol and progesterone; Figs. 1 and 2 , respectively); however, there was no significant difference in RMR between the three test sessions (Fig. 3). This suggests that fluctuations in estradiol and progesterone during the menstrual cycle may not influence RMR. If this is the case the results confirm previous research that suggests RMR can be considered as a stable tool for estimating energy requirement in women ${ }^{(3)}$.

1. Solomon SJ, Kurzer MS \& Calloway DH (1982) Am J Clin Nutr 36, 611-616.

2. Meijer GAL, Westerterp KR, Saris WHM et al. (1992) Am J Clin Nutr 55, 637-640.

3. Soares MJ, Piers LS, Kraai L et al. (1989) Eur J Clin Nutr 43, 465-472. 